\title{
Effect of autologous platelet leukocyte rich plasma injections on atrophied lumbar multifidus muscle in low back pain patients with monosegmental degenerative disc disease
}

\author{
Mohamed Hussein ${ }^{1,}$ and Tamer Hussein ${ }^{2}$ \\ 1 Department of Orthopedics and Traumatology, Surgery New Hospital, Zagazig University Hospitals and Faculty of Medicine, \\ Zagazig University, 44519 Zagazig City, Sharkiah, Egypt \\ 2 Department of Anesthesiology and ICU, Surgery New Hospital, Zagazig University Hospitals and Faculty of Medicine, \\ Zagazig University, 44519 Zagazig City, Sharkiah, Egypt
}

Received 30 May 2015, Accepted 7 January 2016, Published online 22 March 2016

\begin{abstract}
Background: Lumbar multifidus muscle dysfunction and chronic low back pain are strongly correlated. There is no consensus regarding treatment of chronic LBP. The effect of platelet leukocyte rich plasma (PLRP) injections on atrophied lumbar multifidus (LMF) muscle and chronic low back pain has never been studied before. Patients and methods: One hundred fifteen patients with chronic non-specific LBP fulfilled the inclusion criteria. Patients were treated with weekly PLRP injections for six weeks and followed up for 24 months. Primary outcome measures included Numerical Rating Scale (NRS) and Oswestry Disability Index (ODI). Secondary outcome measures included Patient Satisfaction Index (PSI), modified MacNab criteria, and lumbar MRI at 12 months follow-up. Results: One hundred and four patients completed the trial. There were no serious complications. NRS significantly improved gradually from a mean of $8.8 \pm 8$ pre-injection to $3.45 \pm 2.9$ by 12 months and ODI significantly improved gradually from a mean of $36.7 \pm 3.9$ to $14.6 \pm 12.8$ by 12 months $(P<0.005)$. After reaching maximum improvement between 12 and 18 months, all outcome measures remained stable till the end of the 24 months follow-up period with statistically insignificant changes $(P>0.05)$. 87.8\% $(65 / 74)$ of the satisfied patients showed increased crosssectional area and decreased fatty degeneration of LMF muscle on MRI at 12 months follow-up.

Conclusion: PLRP injections into atrophied lumbar multifidus muscle represent a safe, effective method for relieving chronic low back pain and disability with long-term patient satisfaction and success rate of $71.2 \%$. We recommend the use of the lumbar PLRP injections of LMF muscle to refine the inclusion criteria of lumbar fusion to avoid failed back syndrome.
\end{abstract}

Key words: Chronic low back pain, Paraspinal muscles, Atrophied lumbar multifidus muscle, PLRP.

\section{Introduction}

Approximately $70-85 \%$ of all people experience low back pain sometimes in their life [1]. The lumbar multifidus muscle has been proven clinically to be the source of low back pain and referred pain [2]. In healthy individuals, it is believed that the paraspinal muscles, especially the multifidus, play a key role in stabilization of the spine [3]. Atrophy of the paraspinal muscles occurs in chronic LBP with monosegmental degenerative disc disease [4] with reduction in cross-sectional area at L4 and/or L5 [5].

There is no consensus regarding treatment of chronic LBP $[1,6]$. Paravertebral corticosteroids with or without local anesthetics infiltrations have several local and systemic deleterious side effects [7, 8]. However, Back education, physiotherapy with gym-ball exercise program, and perifacet corticosteroid

\footnotetext{
*Corresponding author: m_hussien9@yahoo.com
}

injections may be effective in short-term relief of chronic LBP during the period of treatment [9]. Surgical treatment is controversial and has failed to demonstrate any beneficial effects when compared with nonsurgical treatment or placebo [10]. Moreover, surgery tends to limit motion, thus increasing stresses on adjacent motion segments. Revision lumbar fusion surgery has a clinical failure rate as high as $40 \%$ with nonunion rate of $5-35 \%$ of patients [11].

There are over 1500 proteins within platelets and among them are growth factors stored in platelets as granules, which are known to play important roles in the normal healing response, including PDGF, VEGF, TGF-B, bFGF, EGF, CTGF, and $I G F-1$ [12].

Leukocytes remove tissue debris and they are primarily phagocytic. They secrete growth factors (ILGF) and have a role in balancing the pro-inflammatory and anti-inflammatory aspects of healing [13]. 
(a)

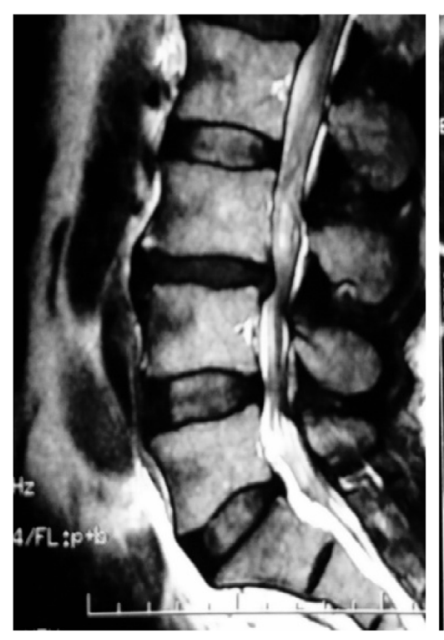

(b)

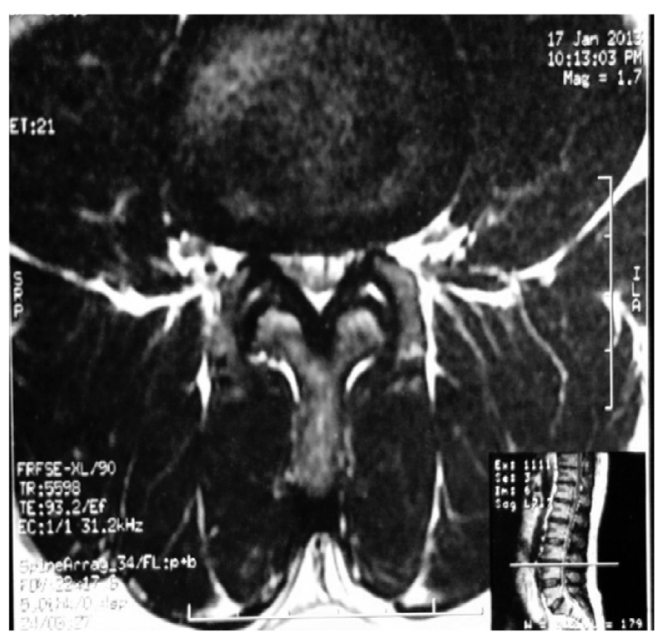

(c)

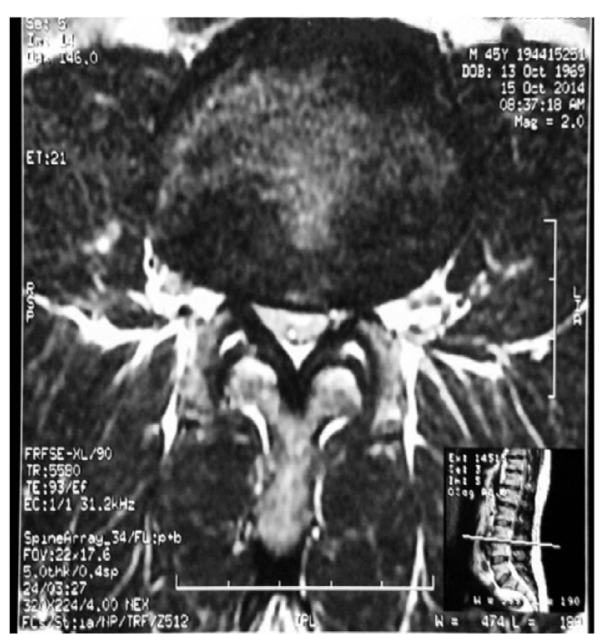

Figure 1. MRI lumbar spine; (a) sagittal view shows L3-4 disc degeneration, (b) axial view of the same patient shows atrophied multifidus on the same level of L3-4 disc degeneration, (c) increased cross-sectional area of LMF after 12 months of local intramuscular PLRP injection.

A review of the literature revealed no clinical studies concerning PLRP injection in atrophied LMF muscle in patients with monosegmental lumbar disc degeneration. We hypothesized that PLRP injection may decrease the chronic LBP and improve disability and quality of life of those patients.

\section{Patients and methods}

One hundred thirty-four patients who complained of chronic LBP with or without nonradicular leg pain continuously for at least three months underwent clinical examination, standardized plain radiography (lateral, oblique, and anteroposterior standing views), and MRI of the lumbar spine at the Orthopedic Department, Zagazig University Hospitals, Egypt. All the patients were assessed clinically and radiographically by two spine surgeons who were not affiliated with the study to determine the inclusion and exclusion criteria. Patients who fulfilled the inclusion criteria were treated with weekly PLRP injection for six weeks into the LMF muscle during the period from May 2012 to October 2012, and then followed up for 24 months to October 2014. All injections were performed by one orthopedic surgeon (M.H.). All the participants gave their written consent in accordance with the Helsinki Declaration [14].

\section{Inclusion criteria}

1. Diffuse unilateral or bilateral continuous LBP for at least three months, with or without leg pain below or above the knee.

2. Moderate (10-50\%) and severe ( $>50 \%)$ LMF muscle atrophy in the MRI scan [15] and one-level degenerative disc disease without disc material extrusion in the canal as shown in the MRI (Figure 1).

LMF muscle atrophy is identified by a decrease in the muscle size and deposition of fat and connective tissue, which shows a high signal intensity on the fast spin echo T2-weighted images used.

\section{Exclusion criteria}

1. Mild changes of less than $10 \%$ of the muscle bulk in the superiomedial part of the LMF muscle as variation of normal [15].

2. Symptomatic spinal stenosis.

3. Disc herniation (extrusion or sequestration) with clear signs of sciatica with positive straight-leg raising (SLR) test.

4. Spondylolysis, spondylolisthesis, and multisegmental degenerative disc disease on the MRI scan.

5. Scoliosis, fracture, previous spine surgery, neuromuscular disease of the trunk, malignancy, infection, and pregnancy.

Thus, after exclusion; the inclusion sample size used in this study consisted of 115 patients and after removing 11 patients who did not complete the injection protocol or the 2-years follow-up period, the final sample size consisted of 104 patients (90.4\% of the included patients). Fifty-six men and 48 women with a mean age of $30.8 \pm 9.21$ years (range 17-55). The mean duration of symptoms was $6.3 \pm 2.4$ months.

\section{Evaluation}

Primary outcome measures included (NRS) (range 0-10) [16] for intensity of pain and ODI [17] - version 2.0 (the sex question [Section 8] is unacceptable in our community, therefore it was removed from the questionnaire). The total possible score became 45 . The final score is calculated and presented as a percentage ( $0 \%$ represents no pain and disability and $100 \%$ represents the worst possible pain and disability). Secondary outcome measures included complication rate, PSI [18], modified MacNab criteria [19], and lumbar MRI done at 12 months follow-up. 


\section{Platelet leukocyte rich plasma preparation}

Fifty milliliters of venous blood sample was collected in sterile tubes containing ACD solution as anticoagulant. The samples were centrifuged twice: the first at $1500 \mathrm{rpm}$ for 15 min. which separates erythrocytes. The second centrifugation at $3000 \mathrm{rpm}$ for $20 \mathrm{~min}$ to concentrate plasma components to produce a unit of $5 \mathrm{~mL}$ of PLRP. One milliliter of the prepared PRP was left for a platelet count, which was done by an automated hemocytometer Cell-Dyn 3700 (Abbott Labs, Dallas, TX, USA). Platelet count ranged $0.8-1.2$ million $/ \mathrm{mL}$. with a mean increase of 4-6-fold of the whole blood values. The prepared amount of PLRP was mixed with $10 \%$ calcium chloride for activation of platelets immediately before injection $(\mathrm{Ca} 2=0.22 \mathrm{~m} \mathrm{Eq} \times$ dose $)$. The amount of PRP prepared is $5 \mathrm{~mL}(10 \%)$ of the amount of the whole blood withdrawn from the patient, following the literature given in references [20-22].

\section{Treatment procedure and follow-up}

After sterile dressing of the skin with Betadine (povidoneiodine), the area was partially anesthetized by spraying ethyl chloride onto the skin. Surgical gloves were worn by the physician during the injections. Injections were performed using a 21-g spinal needle, utilizing the posterior paramedian portal $1.5 \mathrm{~cm}$ laterally from the midline with the patient lying prone over the examination table. Under fluoroscopic guidance the needle is directed vertically $\left(0^{\circ}\right)$ in the axial plane and $5-10^{\circ}$ caudally in the sagittal plane until the tip of the needle hits the lamina of the upper vertebra of the degenerated lumbar motion segment to reach the deep fibers of the LMF muscle. Fix the syringe containing the $5 \mathrm{~mL}$ PLRP to the spinal needle and withdraw the tip of the needle $1-2 \mathrm{~mm}$. Then, inject slowly ( $2.5 \mathrm{~mL}$ per side). During the treatment period, patients are instructed to stop the use of NSAIDs and use cold foments on the lumbar region for pain relief. Patients were advised to walk daily for at least $30 \mathrm{~min}$ and remain as active as possible.

\section{Follow-up}

Follow-up data were obtained from clinic follow-up visits and telephone calls by two independent physicians; before PLRP injections (115 patients), after PLRP injections at day 1 (113 patients in outpatient clinic), 6 months (109 patients: 73 in outpatient clinic and 36 by phone calls), 1 year (105 patients: 67 in outpatient clinic (OPC), 38 by phone calls), and at the final follow-up visit 2 years (104 patients: 67 in OPC, 37 by phone calls) $(90.4 \%)$. The remaining patients were lost for the following reasons: four treatment-unrelated deaths and seven patients who did not respond to telephone calls were excluded from the analysis. Eight patients who underwent intervertebral fusion during the 24 months follow-up period were considered as failure and were included in the analysis.

\section{Statistical analysis}

All statistical analyses were carried out using the SPSS (Statistical Package of Social Sciences, Chicago, IL, USA) for Windows software program version 17.0. $P$ value of less than 0.05 was considered statistically significant. The results were expressed as mean \pm sd. Paired $t$ test and the One-Way ANOVA were used to test for significant differences between baseline band and various follow-up measurements. Chi-square test was used to test the differences between the two groups in terms of categorical data. The General Linear Model (GLM) was used as multivariate analysis to assess the influence of patient characteristics over months of benefit with sex and degree of LMF muscle atrophy as a fixed effect and age and BMI as covariates. Pearson correlation analysis was used to find the correlation between the pre-injection and postinjection measurements for pain and disability.

\section{Results}

There were no serious complications, such as nerve-root injury, multifidus muscle abscess, cauda equina syndrome, spondylodiscitis, or thrombosis. All the patients experienced injection site pain during injection that may last for one or two days. They were prescribed paracetamol or acetaminophen and a muscle relaxant after each PLRP injection to control pain and muscle spasm of the initial inflammatory phase. One hundred and four patients were included in the analysis, 30 patients (28.8\%) did not show any improvement (including the eight patients who underwent lumbar fusion) and the remaining 74 patients $(71.2 \%)$ still had good results without worsening over time at the 24 months follow-up evaluation. The mean duration of the beneficial effects was $18 \pm 6$ months.

The mean NRS back pain score significantly decreased from $8.81 \pm 0.86$ pre-injection, to $3.5 \pm 2.92$ by the end of the 24 months follow-up period with a mean difference of $5.31 \pm 2.90(P>0.001)$. The mean ODI score significantly decreased from $36.74 \pm 3.95$ pre-injection, to $14.65 \pm 12.8$ by the end of the 24 months follow-up period with a mean difference of $22.09 \pm 12.7(P>0.001)$ (Table 1).

The General Linear Model (GLM) showed that the months of benefit were influenced by the degree of LMF muscle atrophy (eta-squared $\eta^{2}=0.567, P=0.00$ ), and not influenced by BMI $\left(\eta^{2}=0.003, P=0.564\right)$, age $\left(\eta^{2}=0.005, P=467\right)$, or sex $\left(\eta^{2}=0.023, P=0.13\right)$ (Table 2). Each term in the model (age, sex, BMI, and LMF muscle atrophy), plus the model as a whole, is tested for its ability to account for variation in the dependent variable (which is the duration of benefit). The significance value for each term, except LMF muscle atrophy, is more than 0.05 . Therefore each term, except LMF muscle atrophy, is statistically insignificant (i.e. has no effect on the duration of benefit).

According to Pearson correlation analysis; there is a strong correlation between the pre-injection and day-1 post-injection measurements for NRS which is statistically significant, indicating that pain level was lower in all patients (i.e. consistent decrease), but for the remaining follow-up measurements, the correlations were weak and they were statistically insignificant indicating that the change was inconsistent across all patients (i.e. several lowered their levels of pain, but several others either did not change or increased their levels). There is a strong correlation between the pre-injection and all the 
Table 1. Mean pre-injection and post-injection NRS (back) and ODI for lumbar multifidus muscle PLRP injection through the follow-up period.

\begin{tabular}{|c|c|c|c|c|c|c|c|}
\hline Outcome & $\begin{array}{l}\text { Pre-inj. } \\
\text { scores }\end{array}$ & Day 1 & 6 months & 12 months & 18 months & 24 months & $\begin{array}{c}\text { Difference in group } \\
\text { means }(95 \% \mathrm{CI})\end{array}$ \\
\hline$N S-1$ & $8.8 \pm(0.86)$ & & & & & & \\
\hline ODI & $73.48 \%(3.95)$ & $63.1 \%(4.5)$ & $29.44 \%(12.8)^{[3]}$ & $29.28 \%(12.8)^{[4]}$ & $29.26 \%(12.8)^{[5]}$ & $29.3 \%(12.8)$ & $44.18 \%(12.7)$ \\
\hline
\end{tabular}

Values are mean standard deviation $(\mathrm{SD}), \mathrm{NRS}=$ Numerical Rating Scale $(0-10)$, ODI $=$ Oswestry Disability Index, CI $=$ Confidence Interval, the mean ODI score is multiplied by two to give the mean disability score which is expressed in the table. Difference in group means is expressed as the difference between pre-injection and post-injection values at the end of follow-up period. The mean ODI and (NRS) back scores for lumbar multifidus PLRP injection showed significant improvement at the end of the 2-years follow-up period, the mean ODI and (NRS) back scores showed statistically insignificant changes and remained low without significant increase again after initial significant improvement that started at the day-1 follow-up that reached maximum improvement by 6-12 months follow-up period post-injection. $P$ (paired $t$ test) $<0.001 *[1] P=0.181$, [2] $P=0.32$, [3] $P=0.083,[4] P=0.741$, [5] $P=0.32$.

Table 2. Explains the practical significance of each variable on the duration of benefit.

\begin{tabular}{|c|c|c|c|c|c|c|}
\hline \multicolumn{7}{|c|}{ Tests of between-subjects effects } \\
\hline \multicolumn{7}{|c|}{ Dependent variable: month of benefit } \\
\hline Source & Type III sum of squares & $d f$ & Mean square & $F$ & Sig. & Partial eta squared \\
\hline$\overline{\text { Corrected model }}$ & $2477.648^{\mathrm{a}}$ & 5 & 495.530 & 48.066 & .000 & .710 \\
\hline Intercept & 154.738 & 1 & 154.738 & 15.010 & .000 & .133 \\
\hline Age & 5.280 & 1 & 5.280 & .512 & .476 & .005 \\
\hline BMI & 3.460 & 1 & 3.460 & .336 & .564 & .003 \\
\hline MF atrophy & 1323.191 & 1 & 1323.191 & 128.349 & .000 & .567 \\
\hline sex & 24.014 & 1 & 24.014 & 2.329 & .130 & .023 \\
\hline MF atrophy $\times$ sex & 10.914 & 1 & 10.914 & 1.059 & .306 & .011 \\
\hline Error & 1010.313 & 98 & 10.309 & & & \\
\hline Total & 16028.000 & 104 & & & & \\
\hline Corrected total & 3487.962 & 103 & & & & \\
\hline
\end{tabular}

${ }^{\mathrm{a}} R^{2}=.710$ (adjusted $\left.R^{2}=.696\right)$. Larger values of partial $\left(\eta^{2}=\right.$ eta squared) indicate a greater amount of variation accounted for by the model term, to a maximum of 1 . Here the individual term (degree of LMF atrophy) is statistically significant and has great effect on the value of months of benefit.

post-injection measurements for ODI score which is statistically significant, indicating that disability level was lower in all patients (i.e. consistent decrease). However, for the day-1 post-injection measurements, the correlation was weak and it was statistically insignificant indicating that the change was inconsistent across all patients. This may be explained by the time needed by the atrophied LMF muscle to regain its strength.

According to modified MacNab criteria, by 18 months post-injection $60.2 \%$ had excellent outcomes, $17.3 \%$ good, $4.0 \%$ fair, and $18.4 \%$ poor; these results remained unchanged throughout the 24 months follow-up period. If the excellent and good categories were regarded as success and fair and poor as failures, then the total success rate was $71.2 \%$ and failure rate was $28.8 \%$ (Figure 2).

According to PSI, $71.2 \%(74 / 104)$ of the patients showed complete satisfaction with the procedure and outcome, and would undergo the injection again for the same condition. While $28.8 \%(30 / 104)$ of the patients were unsatisfied. $87.8 \%$ $(65 / 74)$ of the satisfied patients showed improvement on postinjection MRI done at 12 months of follow-up in the form of increased cross-sectional area and/or decrease in the fat and fibrous tissue content (decrease in high signal intensity on T2-W axial cuts) (Figures 3 and 4).

\section{Discussion}

Our study is the first study that clinically correlates persistent LBP to atrophied LMF muscles by evaluation of the clinical response of LBP patients to PLRP injections of the atrophied LMF muscle. Our preliminary results indicate that chronic LBP is not always due to disc degeneration and instability alone. Also, it proves the role of LMF muscle atrophy as a cause of chronic LBP with or without leg pain. In 2014 Kuittinen et al. [23] did not find any correlation between objective quantitative MRI measures and patient symptoms in degenerative spinal canal stenosis; they suggested other underlying pathobiological mechanisms yet to be discovered.

We suggest a self-sustained vicious cycle which starts with an injury to either the disc, the LMF muscle, or the facet joint. It can lead to further injury to the other two components. Some imaging studies found correlation between chronic LBP and atrophied LMF muscle accompanied by monosegmental degenerative disc disease (two-components) [4, 24]. As long as the injury is limited to one component, the lumbar spine can withstand its loads without pain or disability. Symptomfree people (20-30\%) often have demonstrable lumbar disc degeneration or herniation on MRI $[25,26]$. 


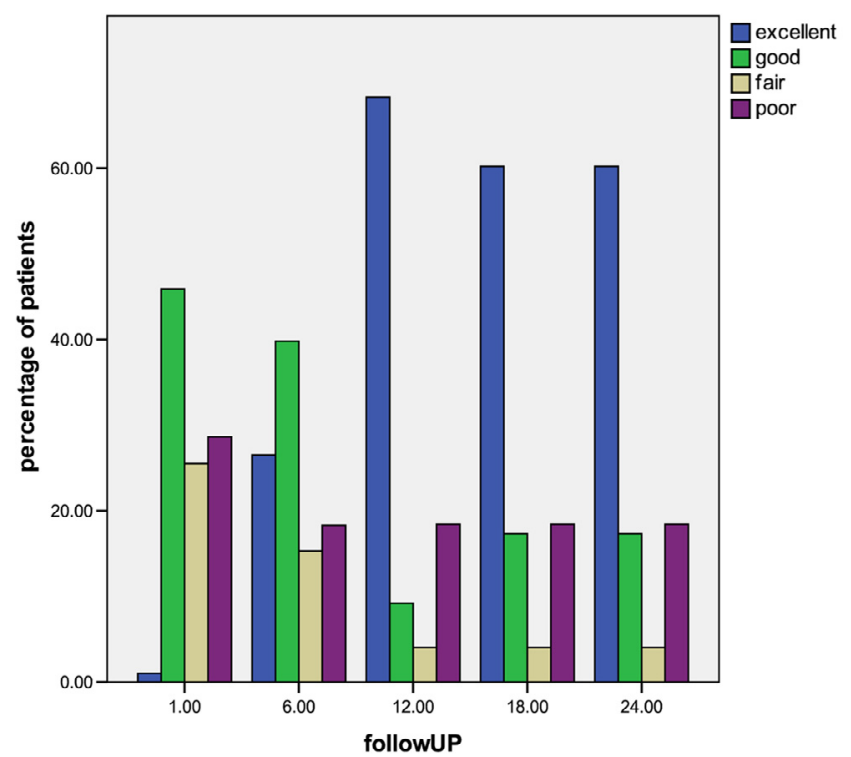

Figure 2. During the first 12 months of the follow-up period the percentage of patients with (excellent) and (good) outcome increased reaching maximum at 12 months follow-up, whereas the percentage of patients with (fair) and (poor) outcome decreased and then remained stable until the end of follow-up.

We observed a short-term reduction in pain after PLRP injections. Platelet concentrates had an analgesic effect [20, 27]. This phenomenon was explained by the presence of large amount of serotonin released from the dense granules of the activated concentrated platelets at the injection site [28]. Overall, the improvement of pain, disability, success rate and patient satisfaction were statistically significant and reached their maximum between 12 and 18 months, then remained stable throughout the follow-up period. This delayed clinical improvement can be explained by time needed by paravertebral neuromuscular tissues to regain its biotensegrity especially the LMF muscle, but proving that it needs further research.

A limitation in our study was the absence of a control group because as mentioned above, there is no consensus (a)

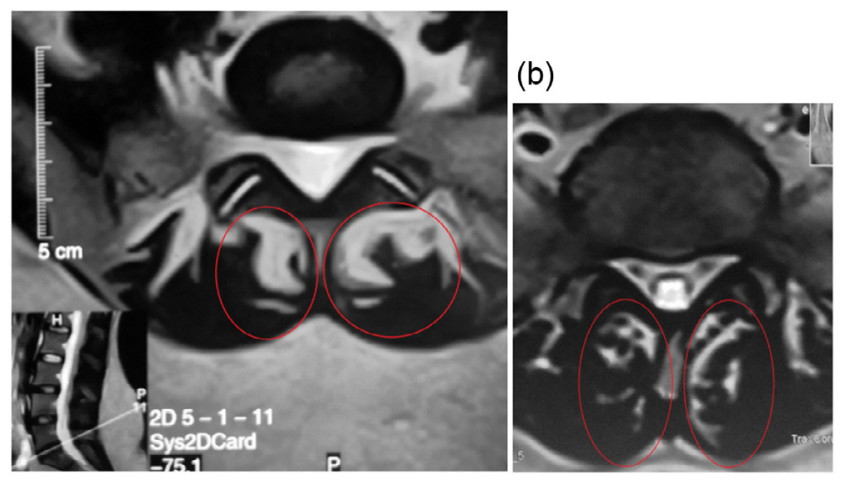

Figure 4. (a) Axial view of lumbar spine MRI shows severe multifidus atrophy, fatty degeneration (high signal intensity) is more than $50 \%$ (red circle), (b) axial view of the same patient after 15 months post-injection of PLRP into atrophied lumbar multifidus with decreased fatty degeneration.

regarding treatment of chronic LBP $[1,6]$. Paravertebral corticosteroids with or without local anesthetics infiltrations are generally performed in the vicinity of nerve roots, even facet infiltration is also a type of nerve-root block, as it is intended to block the posterior branch that emerges directly from the spinal nerve [6-9]. This would create a state of (iatrogenic denervation) increasing the LMF muscle atrophy as denervation leads to atrophy and increased intramuscular fat [29]. Paravertebral corticosteroid infiltrations suppress inflammatory mediators and protein synthesis and suppress hypothalamicpituitary-adrenocortical axis and immunity leading to severe infection [7]. So, we were reluctant to expose the patients in this study to the possibility of corticosteroid side effects for a return of temporary pain relief. We considered this to be unethical.

We used PLRP concentrate of physiological proportions that included concentrated platelets and leukocytes instead of PRP. If platelets are not suspended with biologic levels of other constituents of plasma such as leukocytes, cytokines, and fibrin (the Matrix), the platelet concentrate is either not (a)

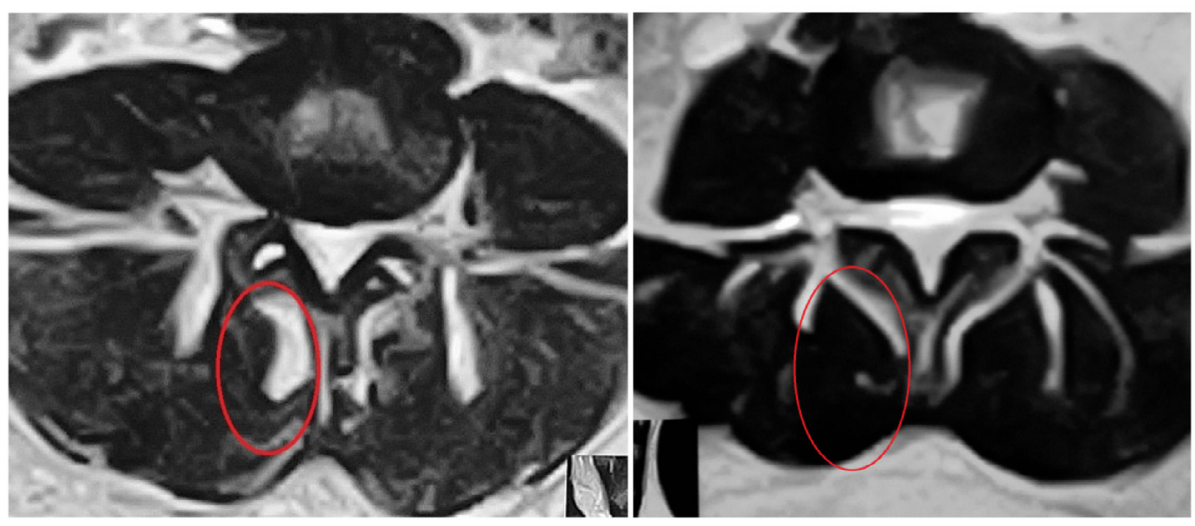

Figure 3. (a) Axial view of lumbar spine MRI shows severe multifidus atrophy, fatty degeneration (high signal intensity) is nearly $50 \%$ (red circle), (b) axial view of the same patient after one year post-injection of PLRP into atrophied lumbar multifidus with decreased fatty degeneration. 
effective or less effective [21]. We had no complications particularly local infection and this may be attributed to the concentrated leukocytes. The density of a leukocyte is approximately the same as a platelet and as a result the procedure also concentrates leukocytes to almost a 10-fold increase. Leukocytes contain some growth factors such as the PDGF$\mathrm{AB}$ that originates from leukocytes in addition to ILGF. Leukocytes have also been shown to produce combinations of proteases and SLPI which inhibits excessive protease activity that leads to destruction of soft tissue and inhibits transition to the proliferative phase of healing [30]. Serhan [31] discovered lipoxins which are proteins secreted by human leukocytes and (LXA4) are formed by 12-lipoxygenase of platelets that depend upon neutrophils for its precursor $\left(\mathrm{LTA}_{4}\right)$. El-Sharkawy and colleagues [32] studied platelet secretions and their effect on macrophage cultures, concluding that PLRP in the 4-6-fold range increases RANTES and Lipoxin A4 (LXA4) which suppresses cytokine release leading to anti-inflammatory effects. Until now, no undesirable inflammatory reactions have been observed with leukocyte rich PRP, even in immune-sensitive applications [13].

We recommend the use of the PLRP injections of atrophied LMF muscle as a safe method that may decrease the chronic LBP and improve disability and quality of life of patients with monosegmental lumbar disc degeneration. We also recommend it as a method to refine the inclusion criteria of lumbar fusion to avoid failed back syndrome.

\section{Abbreviations}

ACD Acid citrate dextrose

bFGF Basic fibroblast growth factor

BMI Body mass index

CTGF Connective tissue growth factor

EGF Epidermal growth factor

GLM General Linear Model

IGF-1 Insulin-like growth factor-1

LBP Low Back Pain

LMF Lumbar Multifidus

LXA4 Lipoxin A4

MD Mean difference

NRS Numerical Rating Scale

NSAIDs Nonsteroidal anti-inflammatory drugs

ODI Oswestry Disability Index

OPC Outpatient clinic

PDGF Platelet derived growth factor

PLRP Platelet leukocyte rich plasma

PRP Platelet rich plasma

RANTES Regulated on activation, normal $\mathrm{T}$ cell expressed and secreted

SLPI Secretory leukocyte protease inhibitor

TGF-B Transforming growth factor beta

VEGF Vascular endothelial growth factor

\section{Conflict of interest}

No external funding was received in support of the present study. No benefits in any form have been or will be received from a commercial party related directly or indirectly to the subject of this manuscript.

M.H. and T.H. certify that they have no financial conflict of interest (e.g., consultancies, stock ownership, equity interest, patent/licensing arrangements, etc.) in connection with this article.

Acknowledgements. The authors acknowledge the assistance of Prof. Dr. Amal Zaidan for her help in PLRP preparation and acknowledge the rest of the Zagazig Orthopedic Department's members (medical and non-medical) for their contributions to the present study.

\section{References}

1. Fritzell P, Hagg O, Wessberg P, Nordwall A (2001) 2001 Volvo award winner in clinical studies: lumbar fusion versus nonsurgical treatment for chronic low back pain: a multicenter randomized controlled trial from the Swedish lumbar spine study group. Spine 26, 2521-2532.

2. Cornwall J, John Harris A, Mercer SR (2006) The lumbar multifidus muscle and patterns of pain. Manual Ther 11(1), $40-45$.

3. Moseley GL, Hodges PW, Gandevia SC (2002) Deep and superficial fibers of the lumbar multifidus muscle are differentially active during voluntary arm movements. Spine 27(2), E29-E36.

4. Ploumis A, Michalidis N, Christodoulou P, Kalaitzoglou I, Gouvas G, Beris A (2011) Ipsilateral atrophy of paraspinal and psoas muscle in unilateral back pain patients with monosegmental degenerative disc disease. Brit J Radiol 84, 709-713.

5. Barker KL, Shamley DR, Jackson D (2004) Changes in the crosssectional area of multifidus and psoas in patients with unilateral back pain: the relationship to pain and disability. Spine 29, E515-E519.

6. Mannion AF, Muntener M, Taimela S et al. (1999) A randomized clinical trial of three active therapies for chronic low back pain. Spine 24, 2435-2448.

7. Holland C, Jaeger L, Smentkowski U, Weber B, Otto C (2012) Septic and aseptic complications of corticosteroid injections: an assessment of 278 cases reviewed by expert commissions and mediation boards from 2005 to 2009. Dtsch Arztebl Int 109(24), 425-430.

8. Krämer J, Blettner M, Hammer GP (2008) Image-guided injection therapy in the lumbar spine. Dtsch Arztebl Int 105(34-35), 596-598.

9. Kader D, Radha S, Smith F, Wardlaw D, Scott N, Rege A, Pope $M$ (2012) Evaluation of perifacet injections and paraspinal muscle rehabilitation in treatment of low back pain. Orthop Traumatol Rehabil 14, 251-259.

10. Gibson JN, Grant IC, Waddell G (1999) The Cochrane review of surgery for lumbar disc prolapse and degenerative lumbar spondylosis. Spine 24, 1820-1832.

11. Hohaus C, Ganey MT, Minkus Y, Meisel JH (2008) Cell transplantation in lumbar spine disc degeneration disease. Eur Spine J 17(Suppl. 4), S492-S503.

12. Qureshi AH, Chaoji V, Maiguel D et al. (2009) Proteomic and phospho-proteomic profile of human platelets in basal, resting state: insights into integrin signaling. PLoS One 4, e7627.

13. Ehrenfest DM, Bielecki T, Del Corso M et al. (2010) Shedding light in the controversial terminology for platelet-rich products: 
platelet-rich plasma (PRP), platelet-rich fibrin (PRF), plateletleukocyte gel (PLG), preparation rich in growth factors (PRGF), classification and commercialism. J Biomedical Mater Res A 95, 1280-1282.

14. World Medical Association. Declaration of Helsinki (Accessed April 30, 2012, at http://www.wma.net/e/policy/b3.htm)

15. Kader DF, Wardlaw D, Smith FW (2000) Correlation between the MRI changes in the lumbar multifidus muscles and leg pain. Clin Rad 55(2), 145-149.

16. Derby R, Howard MW, Grant GM et al. (1999) The ability of pressure controlled discography to predict surgical and nonsurgical outcomes. Spine 24(4), 364-372.

17. Fairbank J, Pynsent P (2000) The Oswestry disability index. Spine 25, 2940-2953.

18. Daltroy LH, Cats-Baril WL, Katz JN et al. (1996) The North American Spine Society (NASS) lumbar spine outcome instrument: reliability and validity tests. Spine 21, 741-749.

19. MacNab I (1971) Negative disc exploration. J Bone Jt Surg Am 53, 891-903

20. Hall MP, Banrd PA, Meislin RJ et al. (2010) Platelet-rich plasma: current concepts and application in sports medicine. J Am Acad Orthop Surg 18(1), 17A.

21. Everts PAM, Knape JTA, van Zundert A et al. (2006) Plateletrich plasma and platelet gel: a review. JECT 38, 174-187.

22. Filardo G, Kon E, Pereira Ruiz MT, et al. (2011) Platelet-rich plasma intra-articular injections for cartilage degeneration and osteoarthritis: single- versus double-spinning approach. Knee Surg Sports Traumatol Arthrosc 20(10), 2082-2091.

23. Kuittinen P, Sipola P, Saari T et al. (2014) Visually assessed severity of lumbar spinal canal stenosis is paradoxically associated with leg pain and objective walking ability. BMC Musculoskelet Disord 15, 348.
24. Kalichman L, Hodges P, Li L, Guermazi A, Hunter D (2010) Changes in paraspinal muscles and their association with low back pain and spinal degeneration: CT study. Eur Spine J 19, 1136-1144.

25. Boden SD, Davis DO, Dina TS, Patronas NJ, Wiesel SW (1990) Abnormal magnetic-resonance scans of the lumbar spine in asymptomatic subjects. A prospective investigation. J Bone Jt Surg Am 72, 403-408.

26. Boos N, Rieder R, Schade V et al. (1995) The diagnostic accuracy of magnetic resonance imaging, work perception, and psychosocial factors in identifying symptomatic disc herniations. Spine 20, 2613.

27. Randelli P, Arrigoni P, Ragone V, Aliprandi A, Cabitza P (2011) Platelet rich plasma in arthroscopic rotator cuff repair: a prospective RCT study, 2-year follow-up. J Shoulder Elbow Surg 20, 518-528.

28. Vanhoutte PM (1991) Platelet-derived serotonin the endothelium and cardiovascular disease. J Cardiol Pharmacol 17S, S6-S12.

29. Dulor JP, Cambon B, Vigneron P et al. (1998) Expression of specific white adipose tissue genes in denervation induced skeletal muscle fatty degeneration. FEBS Lett 439, 89-92.

30. Weibrich G, Kleis W, Hafner G (2002) Growth factor levels in the platelet-rich plasma produced by 2 different methods: curasan-type PRP kit versus PCCS PRP system. Oral Maxillofacial Implants 17, 184-190.

31. Serhan CN (2005) Lipoxins and aspirin-triggered 15-epilipoxins are the first lipid mediators of endogenous antiinflammation and resolution. Prostaglandins Leukot Essent Fatty Acids 73(3-4), 141-162.

32. El-Sharkawy H, Kantarci A, Deady J, Hasturk H, Liu H, Alshahat M, Van Dyke TE (2007) Platelet-rich plasma: growth factors and pro- and anti-inflammatory properties. J Periodontol $78,661-669$.

Cite this article as: Hussein M \& Hussein T (2016) Effect of autologous platelet leukocyte rich plasma injections on atrophied lumbar multifidus muscle in low back pain patients with monosegmental degenerative disc disease. SICOT J, 2, 12 